\title{
Intravenous Thrombolysis in stroke after dabigatran reversal by idarucizumab: our experience and review of literature
}

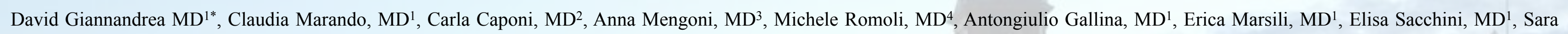
Mastrocola, MD ${ }^{1}$, Chiara Padiglioni, MD ${ }^{1}$, Tatiana Mazzoli, MD ${ }^{1}$, Silvia Cenciarelli, MD ${ }^{1}$, Stefano Ricci, MD ${ }^{1}$.

${ }^{1}$ Neurology Unit - Stroke Unit, Gubbio/Gualdo Tadino and Città di Castello Hospitals, USL Umbria 1, Italy ${ }^{2}$ Internal Medicine Unit, Gubbio/Gualdo Tadino Hospital, USL Umbria 1, Italy

${ }^{3}$ Cardiology and Cardiovascular Physiopathology, S. Maria della Misericordia Hospital, Perugia, Italy

${ }^{4}$ Neurology Clinic, University of Perugia - S. Maria Misericordiae Hospital, Perugia, Italy

Objective: Intravenous thrombolysis (IVT) is contraindicated in case of anticoagulation. Idarucizumab, an antibody specifically binding the direct thrombin inhibitor dabigatran, has been available from October 2015 to promptly reverse anticoagulation. Thereafter, anecdotal cases of IVT after dabigatran reversal with idarucizumab have been reported. Here we describe two cases, and provide a systematic review of all reported cases, defining effectiveness and safety of this treatment paradigm.

Methods: Two patients eligible for IVT for stroke while taking dabigatran were identified from our stroke registry. A systematic review was performed according to PRISMA guidelines and specified criteria (PROSPERO-CRD42017060274). Multivariate analysis was performed to define factors influencing follow-up modified Rankin Scale (mRS).

Results: Including ours, we collected 55 cases, mean age 74.3 years, $43.6 \%$ female. At admission, Median NIHSS was 10 (interquartile range -IQR- 6-14) and mean time from last dabigatran intake was 344 minutes. Clotting studies were abnormal in up to $96 \%$ of cases, with prompt normalization after idarucizumab administration. IVT benefitted 45 patients (81.9\%) (mean time to rtPA 175 minutes), with 5 points median NIHSS improvement (IQR 3-10). Three patients developed symptomatic hemorrhagic transformation, 4 died. An $\mathrm{mRS}<2$ was achieved at follow-up in $56.8 \%$ of patients, but $\mathrm{mRS}$ did not depend on time to IVT or last dabigatran assumption.

Conclusions: Experience with IVT after dabigatran reversal with idarucizumab is limited. However, the treatment paradigm seems safe, and reaches a benefit in the majority of patients receiving it. Larger cohorts are needed to clearly assess bleeding complications and the risk of thrombosis.

Case 1 - A 78-year-old male patient was admitted for a transient ischemic attack occurred the day of admission, characterized by dysarthria and mild right upper limb motor deficit lasting 30 minutes. Personal history revealed type 2 diabetes, dyslipidemia, previous smoking, COPD, 2 previous ischemic strokes, with mild sequelae (mRS 1), and NVAF diagnosed in 2016, for which he was taking dabigatran $110 \mathrm{mg}$ bid.

A few hours after admission the patient developed severe dysarthria, right hemiparesis and confusion (NIHSS 12). Computed tomography (CT) was unremarkable. Trans-cranial-Doppler (TCCD) excluded intracranial vessel occlusion. Lab tests highlighted prothrombin time (PT) 15.2 seconds (normal range: 10-13.5), activated partial thromboplastin time (aPTT) 47 seconds (normal range: 24-35), and international normalized ratio (INR) 1.4. Last dabigatran was taken about 20 hours before. Idarucizumab $5 \mathrm{mg}$ i.v. was given, followed by IVT with $0.9 \mathrm{mg} / \mathrm{kg} \mathrm{rt}-\mathrm{PA}, 180 \mathrm{~min}$ after symptoms onset. Two hours after the end of IVT the NIHSS score dropped to 4 and, after 12 hours only mild dysarthria and postural instability persisted. Control brain MRI excluded haemorrhagic transformation or ischemic lesion demarcation. Carotid ultrasound was unremarkable, while transthoracic echocardiography only revealed atrial dilation. After 6 days the patient restarted oral anticoagulation with apixaban; 90 days functional outcome was good (mRS: 1)

Case 2 - An 85 year-old male with COPD, taking dabigatran $110 \mathrm{mg}$ bid for NVAF, was admitted for a sudden onset of global aphasia and right hemiplegia 3 hours after last dabigatran intake. At admission NIHSS was 22. Head CT scan only showed left middle cerebral artery hyperdensity with no other sign of ischemia. Left MCA occlusion was confirmed with TCCD; carotid ultrasound was unremarkable. Lab test revealed prolonged aPTT (53 sec), with normal prothrombin time and INR. Patient age, premorbid status (mRS 3) and logistic issues did not allow eligibility for endovascular procedures. Thus, 4 hours after symptoms onset, we infused idarucizumab $5 \mathrm{~g}$ i.v., followed by rtPA $0.9 \mathrm{mg} / \mathrm{kg}$. Immediately after the administration of idarucizumab, the whole coagulation panel returned within limits. After thrombolysis, patient status remained unchanged. Control brain CT revealed a left hemispheric ischemic lesion, with deep haemorrhagic infarction. The clinical and neuro-radiological picture remained unchanged for the following 9 days. During the 10th day of hospitalisation, sudden respiratory distress and heart failure led to death.
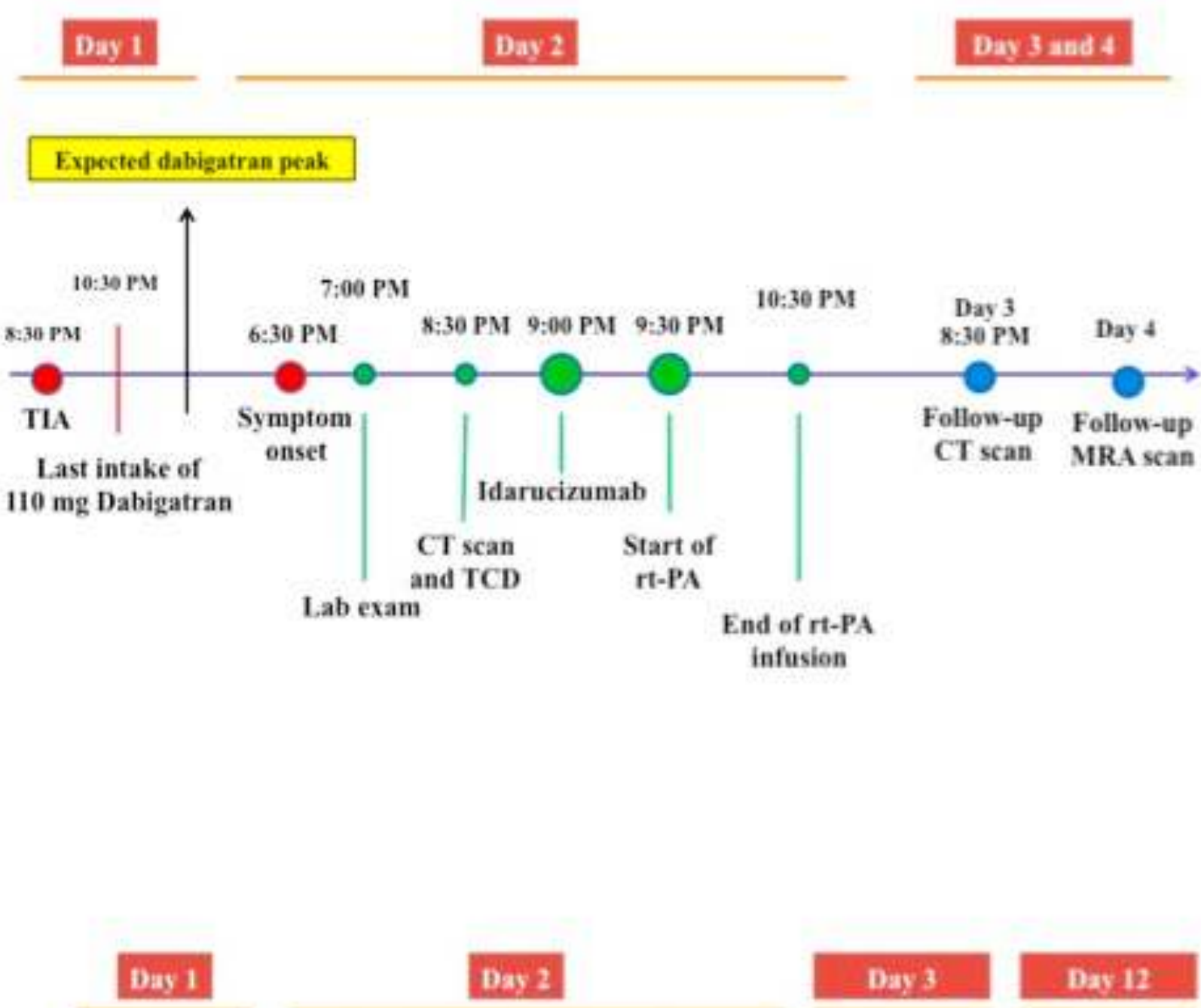

Day 12

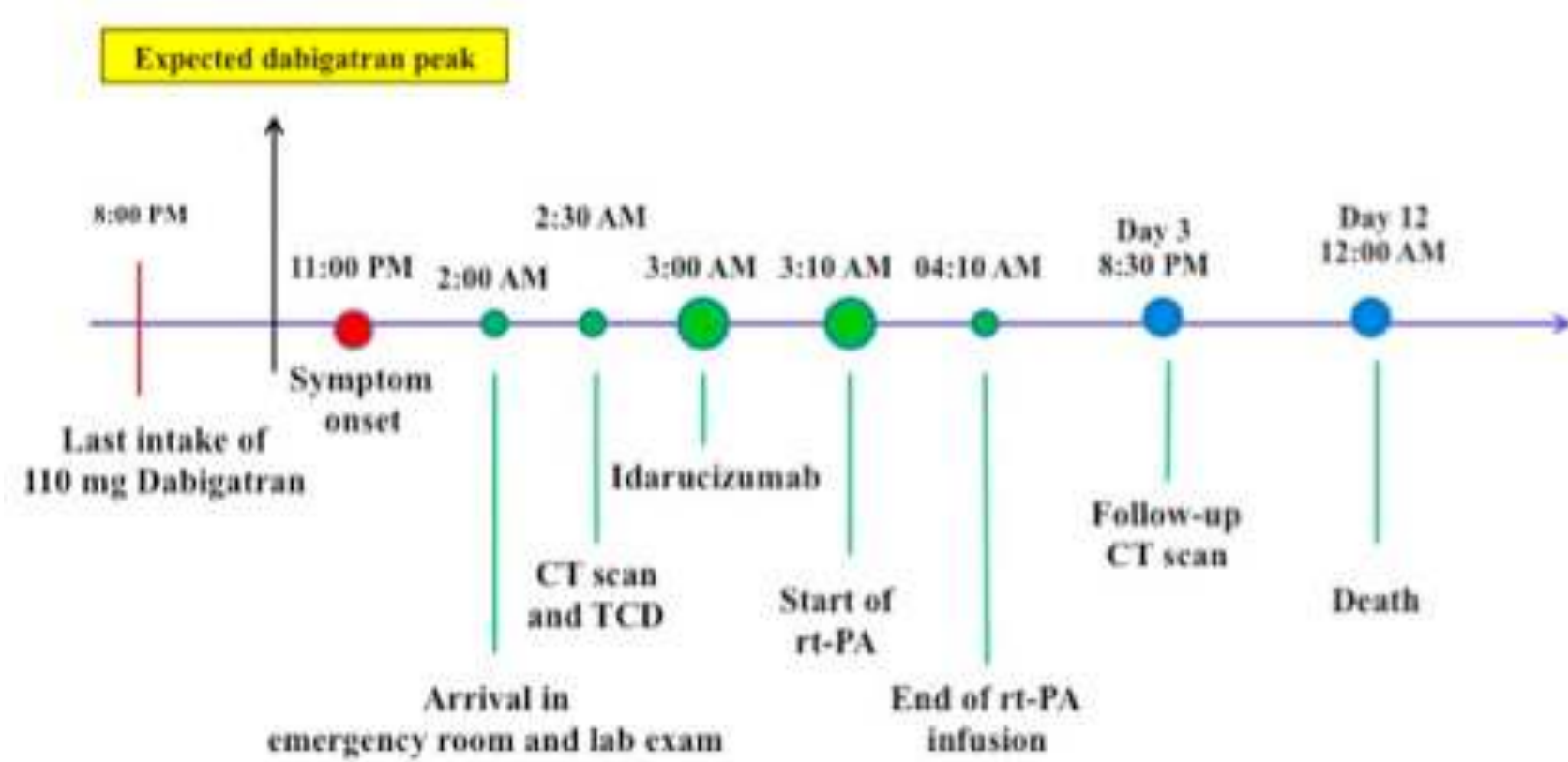

